\title{
National Institute of General Medical Sciences
}

National Cancer Institute

\section{Source}

National Cancer Institute. National Institute of General Medical Sciences. NCI Thesaurus.

Code C20131.

The National Institute of General Medical Sciences (NIGMS) primarily supports basic biomedical research that is not targeted to specific diseases or disorders. 